\title{
The Model for End-Stage Liver Disease-Sodium Score at Admission Is Prognostic of Covid-19 Disease Severity
}

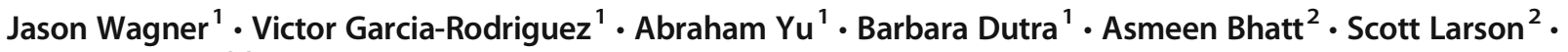 \\ Ahmad Farooq ${ }^{2,3}$
}

Accepted: 17 September 2020 / Published online: 28 September 2020

(C) Springer Nature Switzerland AG 2020

\begin{abstract}
Covid-19 is a systemic viral respiratory illness that can cause gastrointestinal manifestations. There is evidence that Covid-19 can infect liver tissue and may cause transaminemia. A prognostic model is needed to aid clinicians in determining disease severity. The Model for End-Stage Liver Disease-Sodium (MELD-Na) score is a mortality assessment tool in liver transplant patients that has been found to be prognostic in other clinical situations. This study aimed to determine if the MELD-Na score was associated with disease severity in patients with Covid-19, as assessed by multiple clinical outcomes including death within 30 days of discharge and development of an acute kidney injury (AKI). This is a retrospective cohort study that analyzed patients admitted to a community academic hospital with the diagnosis of Covid-19. The 30-day MELD-Na score was found to be significantly higher in those who died $(14.38 \pm 6.92)$ relative to those who survived $(9.68 \pm 5.69 ; p=0.03)$. Additionally, patients with a MELD-Na score greater than 10 were found to have higher risk of developing an AKI (odds ratio (OR) 3.31 (1.08, 10.17); $p=$ 0.03 ), need for hemodialysis (OR 9.69 $(1.74,53.96) ; p=0.007)$, require vasopressors (OR 4.55 $(1.22,16.99) ; p=0.02)$, and have a longer hospital stay (OR $4.17(1.05,16.47) ; p=0.03)$. The MELD-Na score may serve as a useful clinical scoring system for prognosis in patients admitted to the hospital with Covid-19.
\end{abstract}

Keywords Covid-19 $\cdot$ MELD-Na score $\cdot$ Prognostic factor $\cdot$ Acute kidney injury

\section{Introduction}

Covid-19 is a predominantly respiratory illness that can cause systemic symptoms. Prognostic modeling systems are needed to aid clinicians in risk assessment for patients admitted to the hospital with Covid-19. Many have been proposed, however, a recent systematic review has found that few models are reliable [1]. The Model for End Stage Liver Disease-Sodium

This article is part of the Topical Collection on Covid-19

Ahmad Farooq

Ahmad.Farooq@uth.tmc.edu

1 Department of Internal Medicine, University of Texas Health Science Center at Houston, 6431 Fannin, MSB 1.150, Houston, TX 77030, USA

2 Department of Medicine, Division of Gastroenterology, Hepatology and Nutrition, University of Texas Health Science Center at Houston, 6431 Fannin, MSB 1.150, Houston, TX 77030, USA

3 Department of Internal Medicine, Division of Gastroenterology and Hepatology, Duke University, Durham, NC, USA or MELD-Na score is a scoring system that predicts 90-day mortality for patients awaiting liver transplant $[2,3]$. The model has proven to be very useful given its reliance on easily obtained and objective laboratory data. Recently, a study found that the MELD-Na score could accurately predict mortality in patients admitted to the hospital, regardless of the clinical situation [4].

Interestingly, multiple studies have found evidence of liver dysfunction in Covid-19 most commonly manifesting as an elevation in the transaminases $[5,6]$. Recently, pathological and biochemical data has found that the SARS-CoV2 virus can infect liver tissue [7] and may cause inflammation [8]. Given these findings and the usefulness of the MELD-Na score as a prognostic tool, we set out to determine if the MELD-Na score could prognosticate Covid-19 disease severity.

\section{Methods}

This is a retrospective, single-institution, cohort review study conducted in patients admitted with Covid-19 to an academic 
county hospital in Houston, TX, USA. Institutional Review Board approval was granted by the University of Texas Health Science Center at Houston, Houston, TX, USA. Patient information was obtained from an electronic medical record system. Patients were included if they were admitted to the hospital and discharged prior to June 15, 2020, tested positive for Severe Acute Respiratory Syndrome (SARS)-CoV-2 by polymerase chain reaction (PCR)-based testing at least once during admission or if they had a positive diagnosis as an outpatient, and were $\geq 18$ years old. Patients were excluded if they were pregnant, received warfarin at any time during admission, had an active hepatology clinical diagnosis, or had missing data needed to calculate the MELD-Na score.

The primary outcome was mortality based on the Model of End Stage Liver Disease- Sodium (MELD-Na) score. Secondary outcomes included the association of the MELD$\mathrm{Na}$ score and clinical outcomes such as the development of an acute kidney injury (AKI) or need for hemodialysis. Admission laboratory values are the first obtained during the hospital stay (within 48 h of admission). Peak or Nadir laboratory data variables are the highest or lowest obtained during the hospital stay. The deceased within 30-day time point was assessed as follows: patients who were discharged and were not designated as deceased in the EMR within or at 30 days after discharge were presumed to be living. The MELD-Na score was calculated using the online platform provided by the Organ Procurement and Transplantation Network (https:// optn.transplant.hrsa.gov/resources/allocation-calculators/ meld-calculator/). AKI is defined as a rise in serum creatinine by $>0.3 \mathrm{mg} / \mathrm{dL}$ within $48 \mathrm{~h}$.

Data was recorded and analyzed using the computer program Microsoft Excel (Redmond, WA, USA). The computer program R [9] and accompanying R-studio [10] (version 1.2.5033, Orange Blossom) were used for statistical computations. For continuous and categorical data, a Welch twosided $t$ test and a Fisher exact test or Pearson's chi-squared test were used to make comparisons, respectively. For all analyses, a $p$ value $<0.05$ was considered statistically significant. The package epiR [11] was used in the R-studio software to determine odds ratios. Code is available upon request. Missing data was tabulated as NA variables and were excluded from the respective calculation. No funding was used to perform this study.

\section{Results}

In total, 107 patients were admitted to the hospital with Covid19 and discharged prior to June 15, 2020. Five patients were pregnant, two patients received warfarin during admission, and one patient was admitted for acute cholecystitis; these patients were excluded from further analysis. Of note, one patient presented with active hematochezia and one with variceal bleeding; these patients were included in the analysis. The total sample size was 99 (Table 1). The average age was $55.81 \pm 14.05$ and $57 \%$ were male. The median Charlson Comorbidity Index [12] of the population was 2 (IQR: 1-4). Patients were obese with an average BMI of $32.61 \pm 8.33 \mathrm{~kg} /$ $\mathrm{m}^{2}$. A majority of the population $(91 \%)$ had a minority background. Those who died had similar comorbidities relative to those who survived. Of those who died, only $1(6 \%)$ patient had documented liver disease compared with 8 (10\%) in patients who survived at 30 days post-discharge: 1 patient had documented hepatosplenomegaly in the setting of myelofibrosis, 3 patients had alcoholic cirrhosis, 3 patients had hepatic steatosis/fatty liver disease, 1 patient had hepatocellular carcinoma, and 1 patient had cirrhosis secondary to hepatitis C. Nine (10\%) patients had documented hepatitis $\mathrm{C}$ infection and one patient had hepatitis $\mathrm{B}$ infection. Those who died (16.10 7.21 days) had an average length of stay that was significantly longer than those who survived $(9.89 \pm 9.73$ days; $p=$ $0.006)$. Mortality was $16 \%(N=16$ patients $)$.

To assess whether hepatobiliary dysfunction would correlate with disease severity in patients admitted to the hospital with Covid-19, we first assessed if transaminitis was more common in those who died within 30 days of discharge. Although there was a trend towards higher peak transaminases and change in transaminases relative to baseline, no significant differences were found. To evaluate liver function, we compared total bilirubin, albumin, and INR with mortality at discharge. Although non-significant, both the peak total bilirubin and change in total bilirubin relative to baseline were also found to be higher in patients who died relative to those who survived. The highest INR during admission was found to be significantly higher in those who died $(1.66 \pm 0.70, N=$ 16) relative to those who survived $(1.18 \pm 0.30, N=53 ; p=$ $0.01)$. Additionally, admission serum albumin was found to be lower in those who died $(3.19 \pm 0.46, N=16)$ compared with those who survived $(3.47 \pm 0.53, N=75 ; p=0.04)$. Finally, the lowest serum albumin was found to be lower in those who died $(2.37 \pm 0.46, N=16)$ relative to those who survived (3.04 $\pm 0.58, N=76 ; p<0.001)$.

Given the findings that INR and albumin were more likely to be abnormal in patients who died, we applied the MELD$\mathrm{Na}$ model system to determine if it could be of prognostic significance. Data to calculate a MELD-Na score at admission was available for $63(64 \%)$ patients. The MELD-Na score calculated at admission to the hospital was found to be significantly higher in patients who died within 30 days after discharge $(14.38 \pm 6.92, N=13)$ compared with those who survived $(9.68 \pm 5.69, N=50 ; p=0.03)$ (Table 2$)$. To determine a threshold MELD-Na score to use, we studied the association of the MELD-Na score at multiple score thresholds with 30day mortality (Table 3). We found that MELD-Na scores $>10$ and $>15$ were associated with 30 -day mortality by an odds 
Table 1 Basic demographic data for population studied

\begin{tabular}{|c|c|c|c|}
\hline & Total & Deceased within 30 days & Alive at 30 days \\
\hline Sample size & 99 & 16 & 83 \\
\hline Male, count $(\%)$ & $56(57)$ & $13(81)$ & $43(52)$ \\
\hline Age, mean $\pm \mathrm{SD}$, years & $55.81 \pm 14.05$ & $61.37 \pm 13.77$ & $54.73 \pm 13.92$ \\
\hline $\mathrm{BMI}$ mean $\pm \mathrm{SD}, \mathrm{kg} / \mathrm{m}^{2}$ & $32.61 \pm 8.33, N=96$ & $34.17 \pm 10.74, N=15$ & $32.32 \pm 7.86, N=81$ \\
\hline Length of stay, mean \pm SEM, days & $10.89 \pm 9.62$ & $16.10 \pm 7.21$ & $9.89 \pm 9.73$ \\
\hline Charlson Comorbidity Index, mean & $2(1-4)$ & $4(2-6.25)$ & $2(1-4)$ \\
\hline \multicolumn{4}{|l|}{ Race, count $(\%)$} \\
\hline Black/AA & $29(29)$ & $7(44)$ & $22(26)$ \\
\hline Hispanic & $61(62)$ & $8(50)$ & $53(64)$ \\
\hline White/Caucasian & $6(6)$ & $1(6)$ & $5(6)$ \\
\hline Did not identify & $3(3)$ & $0(0)$ & $3(4)$ \\
\hline \multicolumn{4}{|l|}{ Comorbidities, count $(\%)$} \\
\hline Type II diabetes & $48(48)$ & $8(50)$ & $40(48)$ \\
\hline Congestive heart failure & $14(14)$ & $3(19)$ & $11(13)$ \\
\hline Myocardial infarction & $9(9)$ & $2(13)$ & $7(8)$ \\
\hline Chronic kidney disease & 10 & $2(13)$ & $8(10)$ \\
\hline Liver disease & $9(9)$ & $1(6)$ & $8(10)$ \\
\hline COPD & $7(7)$ & $1(6)$ & $6(7)$ \\
\hline Hepatitis C & $9(9)$ & $2(13)$ & $7(8)$ \\
\hline Hepatitis B & $1(1)$ & $1(6)$ & $0(0)$ \\
\hline
\end{tabular}

A Welch two sided $t$ test and a Pearson chi-squared test or fisher exact test were used to make comparisons for continuous and categorical data, respsectively, with $p<0.05$ indicating statistical significance

Sample sizes are indicated next to data point if different from main group heading

$C O P D$, chronic obstructive pulmonary disease; $I C U$, intensive care unit; $S D$, standard deviation

ratio of 9.00 (CI: 2.29, 35.30; $p=0.001$ ) and 5.27 (CI: 1.36, 20.35); $p=0.01$ ), respectively.

Finally, we set out to determine if the MELD-Na score in Covid-19 patients would be associated with clinical outcomes of Covid-19 disease severity (Table 4). Patients with MELDNa scores greater than 10 had an odds ratio of developing an acute kidney injury, need for hemodialysis, need for vasopressors, and length of stay exceeding 7 days by 3.31 (CI: 1.08 , 10.17; $p=0.03$ ), 9.69 (CI: 1.74, 53.96; $p=0.007$ ), 4.55 (CI: $1.22,16.99 ; p=0.02$ ), and 4.17 (CI: $1.05,16.47 ; p=0.03$ ) respectively.

\section{Discussion}

Prognostic markers are needed to aid clinicians in managing Covid-19 patients. The MELD-Na score is a very attractive option, given its simplicity, objectivity, and pervasiveness in clinical medicine. To our knowledge, this is the first time that the MELD-Na score has been applied to Covid-19 patients and found to have prognostic significance. MELD-Na scores greater than 10 were found to be associated with mortality, development of an AKI, need for hemodialysis and vasopressors, and prolonged hospital stay.

The MELD-Na scoring system may capture disease severity in Covid-19 for two reasons. The first is the inclusion of INR into its calculation. It is well known that a coagulopathy occurs in the disease course of Covid-19 illness, which typically manifests as an abnormal elevation in INR $[13,14]$. This coagulopathy has been shown to correlate with mortality [14]. The second reason is the inclusion of creatinine in the MELD$\mathrm{Na}$ score. It is well known that $\mathrm{AKI}$ and need for hemodialysis increase risk of mortality of Covid-19 illness [6, 15, 16]. Interestingly, disease severity models have already proposed incorporate markers of kidney function, such as blood urea nitrogen [17] and glomerular filtration rate [18]. Thus, it is likely that renal function factors into Covid-19 disease severity, which may be captured by the MELD-Na score.

It is interesting that MELD-Na scores of $>10$ and $>20$ appear to have increased risk of death, but scores $>30$ did not. There were few patients in this paper that had scores above 30 (7 patients, 10\%). Thus, it is possible that this study was not powered to detect a difference at this level. More variables will be needed to accurately model disease severity, 
Table 2 The relationship between hepatobiliary laboratory data and mortality

\begin{tabular}{|c|c|c|c|c|}
\hline & Deceased within 30 days, mean $\pm \mathrm{SD}$ & Alive at 30 days, mean $\pm \mathrm{SD}$ & $p$ value & Reference range \\
\hline AST, admission, (U/L) & $38.69 \pm 24.41, N=16$ & $42.24 \pm 28.60, N=76$ & $0.61, \mathrm{~ns}$ & \multirow[t]{3}{*}{$10-40 \mathrm{U} / \mathrm{L}$} \\
\hline AST, peak, (U/L) & $304.20 \pm 751.78, N=15$ & $98.55 \pm 279.11, N=66$ & $0.31, \mathrm{~ns}$ & \\
\hline Change in AST, (U/L) & $390.40 \pm 922.08, N=10$ & $96.89 \pm 340.39, N=44$ & $0.34, \mathrm{~ns}$ & \\
\hline ALT, admission, (U/L) & $26.25 \pm 17.40, N=16$ & $32.49 \pm 23.16, N=76$ & $0.23, \mathrm{~ns}$ & \multirow[t]{3}{*}{$10-40 \mathrm{U} / \mathrm{L}$} \\
\hline ALT, peak, (U/L) & $139.93 \pm 288.28, N=15$ & $78.60 \pm 227.95, N=67$ & $0.45, \mathrm{~ns}$ & \\
\hline Change in ALT, (U/L) & $161.30 \pm 355.86, N=10$ & $67.16 \pm 283.27, N=44$ & $0.44, \mathrm{~ns}$ & \\
\hline $\mathrm{TB}$, admission, (mg/dL) & $0.66 \pm 0.42, N=16$ & $0.94 \pm 2.66, N=76$ & $0.38, \mathrm{~ns}$ & \multirow[t]{3}{*}{$0.3-1.0 \mathrm{mg} / \mathrm{dL}$} \\
\hline $\mathrm{TB}$, peak, $(\mathrm{mg} / \mathrm{dL})$ & $1.37 \pm 1.21, N=15$ & $1.13 \pm 3.30, N=66$ & $0.63, \mathrm{~ns}$ & \\
\hline Change in $\mathrm{TB},(\mathrm{mg} / \mathrm{dL})$ & $0.73 \pm 0.82, N=10$ & $0.21 \pm 0.38, N=43$ & $0.07, \mathrm{~ns}$ & \\
\hline ALP, admission, (U/L) & $73.06 \pm 30.80, N=16$ & $88.02 \pm 42.65, N=76$ & $0.11, \mathrm{~ns}$ & \multirow[t]{3}{*}{$30-120 \mathrm{U} / \mathrm{L}$} \\
\hline ALP, peak, (U/L) & $180.00 \pm 186.16, N=15$ & $108.60 \pm 97.820, N=66$ & $0.16, \mathrm{~ns}$ & \\
\hline Change in ALP, (U/L) & $133.80 \pm 217.00, N=10$ & $34.31 \pm 109.06, N=45$ & $0.18, \mathrm{~ns}$ & \\
\hline Albumin admission, $(\mathrm{g} / \mathrm{dL})$ & $3.19 \pm 0.46, N=16$ & $3.47 \pm 0.53, N=75$ & $0.04 *$ & \multirow[t]{2}{*}{$3.5-5.5 \mathrm{~g} / \mathrm{dL}$} \\
\hline Albumin nadir (gm/dL) & $2.37 \pm 0.46, N=16$ & $3.04 \pm 0.58, N=76$ & $<0.001 *$ & \\
\hline INR admission & $1.15 \pm 0.13, N=13$ & $1.12 \pm 0.26, N=50$ & $0.61, \mathrm{~ns}$ & \multirow[t]{2}{*}{-} \\
\hline INR peak & $1.66 \pm 0.70, N=16$ & $1.18 \pm 0.30, N=53$ & $0.01 *$ & \\
\hline Lipase, admission & $46.33 \pm 77.03, N=6$ & $48.36 \pm 33.80, N=22$ & $0.95, \mathrm{~ns}$ & - \\
\hline
\end{tabular}

Data are presented as mean \pm SEM. A Welch two sided $t$ test was used to make comparisons with $p<0.05$ indicating statistical significance

*Significant result

Sample sizes are indicated next to data point if different from main group heading

$A L T$, alanine aminotransferase; $A L P$, alkaline phosphatase; $A S T$, aspartate aminotransferase; $C I$, confidence interval; ICU, intensive care unit; $I N R$, international normalized ratio; $n s$, non-significant; $S E M$, standard error of the mean; $T B$, total bilirubin

as several have already been proposed [17, 18]. Nevertheless, future prospective studies that have greater sample sizes should be done to determine if higher MELD-Na scores correlate with Covid-19 disease severity.

There are a few conclusions that can be drawn from the analysis of liver function studies analyzed in this paper. The first is that although there were no differences found comparing transaminitis, total bilirubin, and alkaline phosphatase with mortality, the population averages were below the reference upper limit of normal on admission and were mostly above it at their peaks during hospital stay. This may imply that some form of liver damage is occurring during the course of illness. Importantly, this study was not designed to examine liver damage in Covid-19 and thus, future research should compare Covid-19-negative and positive patients to determine if there is truly evidence of liver damage above what is usually seen in hospitalized patients. Additionally, this study was not controlled for use of medications, other than warfarin, which can impact liver function.

Limitations of this study include a smaller sample size, focus on one community hospital, and the retrospective nature of the study, precluding causative analysis. Strengths include analysis of a predominantly minority population, analysis of a population that was followed post-discharge, and reliance on objective clinical data to determine prognosis. Taken together, we find here that the MELD-Na score may serve as a useful prognostic numerical assessment of disease severity for patients admitted to the hospital for Covid-19.
Table 3 The MELD-Na score is higher in patients with Covid-19 who died at 30 days postdischarge relative to those who survived

\begin{tabular}{llll}
\hline & $\begin{array}{l}\text { Deceased within } \\
30 \text { days, mean } \pm \mathrm{SD}\end{array}$ & $\begin{array}{l}\text { Alive at 30 days, } \\
\text { mean } \pm \mathrm{SD}\end{array}$ & Significance \\
\hline Sample size & 13 & 50 & - \\
Sodium (mEq/L), admission & $134.85 \pm 4.69$ & $136.62 \pm 4.11$ & $0.22, \mathrm{~ns}$ \\
Creatinine (mg/dL), admission & $2.40 \pm 3.00$ & $1.63 \pm 2.65$ & $0.40, \mathrm{~ns}$ \\
Total Bilirubin (mg/dL), admission & $0.64 \pm 0.41$ & $1.08 \pm 3.27$ & $0.36, \mathrm{~ns}$ \\
INR, admission & $1.15 \pm 0.13$ & $1.12 \pm 0.26$ & $0.61, \mathrm{~ns}$ \\
MELD-Na score, admission & $14.38 \pm 6.92$ & $9.68 \pm 5.69$ & $0.03^{*}$ \\
\hline
\end{tabular}

Data are presented as mean \pm standard deviation

*Significance calculated using a two-sided Welch $t$ test, with a $p<0.05$ indicating significance

$I N R$, international normalized ratio; $M E L D$, model of end-stage liver disease; $N a$, sodium; $\mathrm{S} D$, standard deviation 
Table 4 The relationship between the MELD-Na > 10 and clinical outcomes of Covid-19 disease severity

\begin{tabular}{|c|c|c|c|c|}
\hline & MELD-Na $>10$, count $(\%)$ & MELD-Na $<10$, count $(\%)$ & Odds ratio & Significance \\
\hline Sample Size & 19 & 44 & - & - \\
\hline Need for ICU Admission & $10(53)$ & $17(39)$ & $1.76(0.60,5.23)$ & $0.30, \mathrm{~ns}$ \\
\hline Develop $\mathrm{AKI}^{1}$ & $12(63)$ & $15(34)$ & $3.31(1.08,10.17)$ & $0.03 *$ \\
\hline Need Supplemental oxygen & $16(84)$ & $39(89)$ & $0.68(0.15,3.21)$ & $0.63, \mathrm{~ns}$ \\
\hline Need intubation & $7(37)$ & $8(18)$ & $2.63(0.79,8.77)$ & $0.11, \mathrm{~ns}$ \\
\hline Need for vasopressors & $7(37)$ & $5(11)$ & $4.55(1.22,16.99)$ & $0.02 *$ \\
\hline Length of stay $>7$ days & $15(83)$ & $24(55)$ & $4.17(1.05,16.47)$ & $0.03 *$ \\
\hline Need for Hemodialysis & $6(32)$ & $2(5)$ & $9.69(1.74,53.96)$ & $\ddagger_{0.007 *}$ \\
\hline
\end{tabular}

Data are presented as count (percentage of total sample size). Pearson's chi-squared test was used to make comparisons with $p<0.05$ indicating statistical significance

*Significant result

$M E L D$, model of end-stage liver disease; $N a$, sodium; $n s$, non-significant

${ }^{1}$ AKI: defined as a rise in serum creatinine $>0.3 \mathrm{mg} / \mathrm{dL}$ within $48 \mathrm{~h}$

*Fisher's exact test was used to determine significance

Authors' Contributions JW, VR, AY, BD, and AF were involved in planning the study. JW, VR, AY, and BD collected data. JW, VR, AY, $\mathrm{AB}, \mathrm{SL}$, and AF performed data analysis and wrote the manuscript. All authors have approved of the final draft.

\section{Compliance with Ethical Standards}

Conflict of Interest The authors declare that they have no conflict of interest.

Ethical Approval All procedures performed in studies involving human participants were in accordance with the ethical standards of the institutional and/or national research committee and with the 1964 Helsinki declaration and its later amendments or comparable ethical standards.

Informed Consent Informed consent was obtained from all individual participants included in the study.

\section{References}

1. Wynants L, Van Calster B, Collins GS, et al. Prediction models for diagnosis and prognosis of covid-19 infection: systematic review and critical appraisal. BMJ. 2020;369:m1328.

2. Kim WR, Biggins SW, Kremers WK, et al. Hyponatremia and mortality among patients on the liver-transplant waiting list. $\mathrm{N}$ Engl J Med. 2008;359:1018-26.

3. Malinchoc M, Kamath PS, Gordon FD, Peine CJ, Rank J, ter Borg PC. A model to predict poor survival in patients undergoing transjugular intrahepatic portosystemic shunts. Hepatology. 2000;31:864-71.

4. Roth JA, Chrobak C, Schadelin S, Hug BL. MELD score as a predictor of mortality, length of hospital stay, and disease burden: a single-center retrospective study in 39,323 inpatients. Medicine (Baltimore). 2017;96:e7155.

5. Guan WJ, Ni ZY, Hu Y, et al. Clinical characteristics of coronavirus disease 2019 in China. N Engl J Med. 2020;382:1708-20.

6. Zhou F, Yu T, Du R et al. Clinical course and risk factors for mortality of adult inpatients with COVID-19 in Wuhan, China: a retrospective cohort study Lancet 2020;395:1054-1062.
7. Puelles VG, Lutgehetmann M, Lindenmeyer MT et al. Multiorgan and renal tropism of SARS-CoV-2. N Engl J Med. 2020.

8. Xu Z, Shi L, Wang Y, et al. Pathological findings of COVID-19 associated with acute respiratory distress syndrome. Lancet Respir Med. 2020;8:420-2.

9. R Core Team (2020). R: a language and environment for statistical computing. R Foundation for statistical computing V, Austria. URL, https://www.R-project.org/.

10. RStudio T. RStudio: Integrated Development for R. RStudio, PBC, Boston, MA URL http://www.rstudio.com/. City;2020.

11. Mark Stevenson TN, Cord Heuer, Jonathon Marshall, Javier Sanchez, Ron Thorn-ton, Jeno Reiczigel, Jim Robison-Cox, Paola Sebastiani, Peter Solymos, Kazuki Yoshida, Ge-off Jones, Sarah Pirikahu, Simon Firestone, Ryan Kyle, Jo-hann Popp, Mathew Jay and Charles Reynard. EpiR: Tools for the analysis of epidemiological data. City;2020.

12. Charlson ME, Pompei P, Ales KL, MacKenzie CR. A new method of classifying prognostic comorbidity in longitudinal studies: development and validation. J Chronic Dis. 1987;40:373-83.

13. Connors JM, Levy JH. COVID-19 and its implications for thrombosis and anticoagulation. Blood. 2020;135:2033-40.

14. Tang N, Li D, Wang X, Sun Z. Abnormal coagulation parameters are associated with poor prognosis in patients with novel coronavirus pneumonia. J Thromb Haemost. 2020;18:844-7.

15. Cheng Y, Luo R, Wang K, et al. Kidney disease is associated with inhospital death of patients with COVID-19. Kidney Int. 2020;97:829 38.

16. Ali H, Daoud A, Mohamed MM, et al. Survival rate in acute kidney injury superimposed COVID-19 patients: a systematic review and meta-analysis. Ren Fail. 2020;42:393-7.

17. Gong J, Ou J, Qiu X, et al. A tool for early prediction of severe coronavirus disease 2019 (COVID-19): a multicenter study using the risk nomogram in Wuhan and Guangdong, China. Clin Infect Dis. 2020;71:833-40.

18. Zhang C, Qin L, Li K, et al. A novel scoring system for prediction of disease severity in COVID-19. Front Cell Infect Microbiol. 2020;10:318.

Publisher's Note Springer Nature remains neutral with regard to jurisdictional claims in published maps and institutional affiliations. 